\title{
Lógica borrosa como herramienta de apoyo en la elaboración de un presupuesto base cero: Caso artesanos de muebles de madera.
}

\author{
Fuzzy logic as a support tool in the elaboration of a zero base budget: Case \\ wood furniture craftsmen
}

Juan Ennis Espinoza González. ${ }^{1}$, Kléber Antonio Luna Altamirano. ${ }^{2}$, William Henry Sarmiento Espinoza. ${ }^{3}$ \& Juan Carlos Erazo Álvarez. ${ }^{4}$

Recibido: 19-02-2019 / Revisado: 26-02-209 /Aceptado: 24-03-2019/ Publicado: 05-04-2019

\begin{abstract}
.
DOI: https://doi.org/10.33262/cienciadigital.v9i2.411

The present article refers to the structure of a budget based on a new technique of analysis for artisans in the manufacture of wooden furniture in the canton Santiago de Méndez-Ecuador, of the Province of Morona Santiago, called Zero Base Budget (PBC), since the problem lies in the ignorance of the administrators of these companies at the time of constructing a budget. The objective of this study is to give these artisans a new way to prepare budgets based on objectives and goals set by these organizations, the methodology used is based on the support of advanced tools that offers fuzzy logic, such as trapezoidal blur numbers (NBTr), which traps correct budget values in order to reduce uncertainty at the time of making projections into the future. As a result of this research is to achieve that budget levels are ideal and achievable in order to improve management in these craft enterprises.
\end{abstract}

Keywords: Fuzzy Logic, Zero Base Budget, Trapezoidal Fuzzy Numbers

\section{Resumen.}

1 Universidad Católica de Cuenca, Maestrante en Administración de Empresas, Cuenca, Ecuador. jespinozag@psg.ucacue.edu.ec

2 Universidad Católica de Cuenca, Maestrante en Administración de Empresas, Cuenca, Ecuador. klunaa@ucacue.edu.ec

3 Universidad Católica de Cuenca, Maestrante en Administración de Empresas, Cuenca, Ecuador. wsarmiento@ucacue.edu.ec

${ }^{4}$ Universidad Católica de Cuenca, Posgrados, Cuenca, Ecuador. jcerazo@ucacue.edu.ec 
El presente artículo, se refiere a la estructura de un presupuesto basado en una técnica nueva de análisis para los artesanos en la fabricación de muebles de madera del cantón Santiago de Méndez de la Provincia de Morona Santiago-Ecuador, denominado Presupuesto Base Cero (PBC), ya que el problema radica en el desconocimiento de los administradores de estas empresas al momento de construir un presupuesto. El objetivo de este estudio es entregar a estos artesanos una nueva forma de elaborar presupuestos basado en objetivos y metas trazados por estas organizaciones, la metodología utilizada se basa en el apoyo de herramientas de avanzada que ofrece la lógica difusa, como son los números borrosos trapezoidales (NBTr), en donde se atrapa valores correctos presupuestarios con la finalidad de reducir la incertidumbre en el momento de realizar proyecciones a futuro. Como resultado de esta investigación es lograr conseguir que los niveles presupuestarios sean ideales y alcanzables con el propósito de mejorar la gestión en estas empresas artesanales.

Palabras Clave: Lógica difusa, presupuesto base cero, números borrosos trapezoidales

\section{Introducción.}

El cantón Santiago de Méndez de la Provincia de Morona Santiago-Ecuador, eminentemente productor de madera, en donde sus artesanos fabrican muebles de hogar con esta materia prima, acrecentando el desarrollo tanto local, regional y nacional. El problema de este estudio, radica en el desconocimiento por parte de los administradores de estas empresas en como estructurar un presupuesto, realizando de una manera empírica, únicamente basándose en la experiencia y en un historial del año anterior, para posteriormente proyectarse de acuerdo a las necesidades de la empresa, el cual arrastra vacíos presupuestarios y estos se extrapolan al futuro.

El objetivo de la investigación, es aportar con nuevo conocimiento a aquellos artesanos quienes se dedican a la fabricación de muebles de madera, para una nueva forma de elaborar presupuestos, este se basa en el cumplimento de objetivos trazados (PBC), estos inconvenientes se van eliminando al agregar como principio la justificación a cualquier gasto que se pretenda dar en el futuro, partiendo de cero y no de valores de los años pasados, y con el apoyo de la lógica difusa con aplicación de herramienta de avanzada como los números borrosos trapezoidales (NBTr), se tratará de reducir la incertidumbre en la toma de decisiones a nivel de gerencia.

Los presupuestos representan el concepto cuantitativo cabal dentro de un período que la empresa desea alcanzar sus objetivos trazados, mediante el desarrollo de estrategias esenciales para lograrlo (Burbano 2005). El Presupuesto Base Cero inicia como una idea para la estructura de un modelo presupuestario en la empresa Texas Instruments, que luego se propague a otras organizaciones por el éxito de este método. La parte importante en la 
elaboración de este tipo de presupuesto se basa en una correcta planificación y cumplimiento de sus objetivos conllevando a una eficiente gestión empresarial.

En este artículo, se explica cómo elaborar un Presupuesto Base Cero con lógica borrosa apoyados en números borrosos trapezoidales (NBTr), representando valores dentro los cuales puede ocurrir un determinado suceso. Estos números se expresan en cuádruplos, donde el número del lado izquierdo significa el valor mínimo y el número de lado derecho representa el valor máximo alcanzado, en cambio los dos números centrales constituyen los valores más posibles que puedan suceder el acontecimiento. Lazzari (1997) explica que un (NBTr) está expresado por cuatro números reales (el valor mínimo, el valor máximo y los valores de mayor nivel de presunción), los (NBTr) representarán la opinión de los expertos en un rango más amplio.

Dentro de la metodología, se explica de madera detallada la aplicación de instrumentos que ofrece la lógica difusa conocida también como lógica borrosa, para recopilar información y elaborar un presupuesto base cero. Luego se procede a explicar que áreas son de interés dentro de la empresa fabricante de muebles de madera, y su respectiva evaluación por los expertos conocedores del tema. Finalmente se realiza los cálculos utilizando números borrosos trapezoidales (NBTr) y se presenta los resultados correspondientes.

\section{Estado del arte}

En la actualidad la sociedad se enfrenta a cambios dentro del ámbito financiero, por ello se ha ido apartando de la utilización de instrumentos lineales en la elaboración de presupuestos los cuales conllevaban a sesgar los resultados, por ello algunos autores dan a conocer la aplicación de este nuevo modelo de elaboración de presupuestos, entre ellos: Ciscomani (2015) propone un nuevo esquema de gestión pública, a partir de la aplicación de un presupuesto base cero, una nueva visión estratégica para la gestión e innovación administrativa, tiene como propósito principal articular una política interinstitucional que permita alcanzar los objetivos de la política pública en los diversos sectores, de manera equilibrada y armónica. Tovar (2015) explica que el presupuesto base cero se deriva de la opinión de que ningún egreso está estimado como dado, todo presupuesto se ajusta a un proceso de análisis y se eliminan las inercias de presupuestos realizados en ejercicios anteriores. Sánchez (2015) indica que México enfrenta hoy una situación económica que hace indispensable elaborar un Presupuesto Base Cero, este consiste en establecer esquemas de planeación que se aplican tanto en la gestión empresarial como en los programas públicos. Pérez (2015) realiza un análisis entre el Presupuesto Base Cero y el Presupuesto basado en Resultados, partiendo que el primero (PBC) puede ser de utilidad en la presupuestación de gastos operativos y administrativos de gobierno, en cambio el $(\mathrm{PbR})$ es más efectivo para la presupuestación de programas sustantivos de gobierno que impacten en el ciudadano. Chávez (2015) sostiene: "Presupuesto Base Cero (PB0) es un concepto, al que se recurre para 
determinar un nuevo nivel de asignación de recursos a una organización, (...), sin tomar en cuenta la asignación presupuestaria en curso o la que imperó en el pasado" (p.70).

Dentro de la gestión empresarial, la elaboración de un Presupuesto Base Cero con apoyo de la lógica difusa, se convierte en una herramienta poderosa para la toma de decisiones. En 1965 Lotfi Asker Zadeh adopta conceptos de la lógica y de los subconjuntos borrosos, por medio de la definición de grados de pertenencia, dando los inicios para el nacimiento de la lógica difusa. Otros autores dan a conocer la aplicación de herramientas de avanzada que ofrece la lógica borrosa, con desarrollo de la metodología "Fuzzy Set", en la solución de problemas a nivel empresarial, entre ellos: Kaufmann y Gil (1986). Introducción de la teoría de subconjuntos borrosos a la gestión de las empresas; Kaufmann y Gil (1987). Técnicas operativas de gestión para el tratamiento de la incertidumbre; Lafuente (1997). Marketing para el nuevo milenio: nuevas técnicas para la gestión comercial en la incertidumbre; Arango, Urán y Pérez (2012). La gestión de indicadores empresariales con lógica difusa para la toma de decisiones; Chalco et al. (2009). Una aproximación de números difusos de tipo trapezoidal; Medina (2006). Estado de la cuestión acerca del uso de la lógica difusa en problemas financieros; Luna et al. (2018). Implementación de un presupuesto empresarial base cero bajo el enfoque difuso; Reig y González (2002) afirman: "la lógica borrosa se revela como un instrumento muy potente (...) al permitir, por un lado, recoger la incertidumbre generada por el entorno de la empresa, y por otro tratar la subjetividad que implica toda opinión de expertos" (p.436).

Los autores antes mencionados, mediante el apoyo de la lógica difusa han demostrado la eficacia de esta teoría, en donde se trata de reducir la incertidumbre en la información con el propósito de mejorar la gestión empresarial y que la toma de decisión sea la correcta.

\section{Metodología}

El presupuesto Base cero ( $\mathrm{PBC}$ ), parte de objetivos que la empresa se plantea y de acuerdo a ello se entrega los recursos a las diferentes áreas de la organización, con el propósito de que se llegue a cumplir con las metas trazadas. Con el apoyo de la lógica difusa se trata de reducir la incertidumbre en la elaboración de un presupuesto que considere los objetivos planteados para una empresa fabricante de muebles de madera en el cantón Santiago de Méndez de la Provincia de Morona Santiago, esperando su posible cumplimiento, con ello se aporta a una eficiente gestión empresarial. La aplicación de números borrosos trapezoidales (NBTr) ayuda a realizar predicciones a futuro, se determina cuatro áreas o departamentos y doce objetivos que se pretende alcanzar en los siguientes periodos.

Se presenta a modo de ejemplo los pasos para la elaboración de un Presupuesto Base Cero (PBC), con aplicación de números borrosos trapezoidales que ofrece la lógica borrosa. Primero se determina las áreas de trabajo, se establecen los objetivos a cumplir, y sus 
respecticos rubros económicos establecidos en un tiempo determinado. Lo enunciado se detalla en la siguiente tabla.

Tabla 1. Áreas de trabajo

AREAS OBJETIVOS

UNIDAD MONETARIA

A: Comercialización

$\mathrm{A} 0=$ Incrementar o aumentar las ventas

A1 = Mejorar servicio de rentas

2.000,00 dólares

800,00 dólares

B: Producción

B0 = Selección y capacitación de mano de obra

3.500,00 dólares calificada

B1 = Crear modelos innovadores

B2 $=$ Mejorar la productividad

800,00 dólares

500,00 dólares

C: Marketing

$\mathrm{C} 0=$ Mejorar publicidad para competir en e

500,00 dólares mercado

$\mathrm{C} 1=$ Encontrar un nicho de mercado

1.700,00 dólares

$\mathrm{C} 2=$ Crear un plan de marketing

1.000,00 dólares

D: Administración

D0 = Mejorar los equipos de cómputo

1.500,00 dólares

D1 = Implementación de internet y software

500,00 dólares

$\mathrm{D} 2=$ Implementación de muebles y 1.000,00 dólares decoraciones

\section{Fuente: Elaboración propia}

Como segundo paso, se determina los ingresos generados en un determinado periodo, estos datos se presentan en la siguiente tabla.

Tabla 2. Ingresos Estimados

\begin{tabular}{|l|lc|}
\hline Ventas por unidades al año & $\$$ & $150.500,00$ \\
Cobros a corto plazo & $\$$ & $46.500,00$ \\
Cobros a largo plazo & $\$$ & $23.500,00$ \\
Otros Ingresos & $\$$ & $1.200,00$ \\
\hline INGRESOS TOTALES ESTIMADOS & $\$$ & $221.700,00$ \\
\hline
\end{tabular}

Fuente: Elaboración propia 
Se totaliza los objetivos predispuestos a cumplir por la organización, se presenta en el presupuesto, mediante niveles para cada una de las áreas.

Tabla 3. Cifra Presupuestaria

\begin{tabular}{|l|ll|}
\hline $\mathrm{A}=$ COMERCIALIZAC IÓN & $\$$ & $2.800,00$ \\
$\mathrm{~B}=$ PRODUCCIÓN & $\$$ & $4.800,00$ \\
$\mathrm{C}=$ MARKETING & $\$$ & $3.200,00$ \\
$\mathrm{D}=$ ADMINISTRACIÓN & $\$$ & $3.000,00$ \\
\hline TOTAL & $\$$ & $13.800,00$ \\
\hline
\end{tabular}

Fuente: Elaboración propia

Kaufmann y Gil-Aluja (1989) afirman: "La introducción de una valuación matizada entre 0 y 1 permite hacer intervenir niveles de verdad en la noción de incidencia. (...) Valores de 0 a 1 (la llamada valuación endecadaria)" (p. 26). El principio de la Simultaneidad Gradual, explicado por Gil Aluja (1986) establece que cualquier proposición puede ser verdadera y falsa al mismo tiempo, siempre y cuando se le dé un grado de verdad y un grado de falsedad. La escala endecadaria utilizada para este análisis se presenta en la siguiente tabla.

Tabla 4. Escala endecadaria

\begin{tabular}{ll}
\hline GRADO DE & DRESUNCIÓN $\alpha$ \\
\hline 0 & INCIDENCIA \\
0,1 & Bajo \\
0,2 & Prácticamente bajo \\
0,3 & Muy bajo \\
0,4 & Bastante Bajo \\
0,5 & Más bajo que alto \\
0,6 & Tan bajo como alto \\
0,7 & Más alto que bajo \\
0,8 & Bastante alto \\
0,9 & Muy alto \\
1 & Prácticamente alto \\
\hline
\end{tabular}

Fuente: Elaboración propia

Se recurre a la escala endecadaria (tabla 4) con el propósito de obtener información de los expertos, en este caso los administradores y el personal responsable de la parte financiera de la fábrica de muebles de madera. A nivel de administración, ésta es la responsable de establecer valores monetarios con la finalidad de dar cumplimiento a los objetivos trazados, 
para ello se determina 9.170,00 dólares, en el caso mínimo; y 11. 880,00 dólares en el caso máximo, estos valores se expresan en la tabla 5.

Tabla 5. Recursos económicos para el cumplimiento de objetivos

\begin{tabular}{|l|lr|lr|}
\hline Componentes de los Ingresos & \multicolumn{2}{|l|}{$\begin{array}{l}\text { Posición } \\
\text { Pesimista }\end{array}$} & \multicolumn{2}{l|}{ Posición } \\
& Optimista \\
\hline Ventas por unidad & $\$$ & $5.250,00$ & $\$$ & $6.500,00$ \\
Cobros a corto plazo & $\$$ & $2.100,00$ & $\$$ & $2.950,00$ \\
Cobros a largo plazo & $\$$ & $1.300,00$ & $\$$ & $1.830,00$ \\
Otros Ingresos & $\$$ & 520,00 & $\$$ & 600,00 \\
\hline TOTALES & $\$$ & $9.170,00$ & $\$$ & $11.880,00$ \\
\hline
\end{tabular}

Fuente: Elaboración propia

En el siguiente gráfico, se observa los valores de la restricción presupuestaria en unidades de mil, la empresa tratará de dar cumplimiento con los objetivos establecidos (posición optimista). Con relación a la posición pesimista, este se puede llegar con mayor seguridad, el cual toma una estimación de uno frente a la otra, cuyo valor será de cero si se supera dicho monto. Lo manifestado se detalla a continuación.

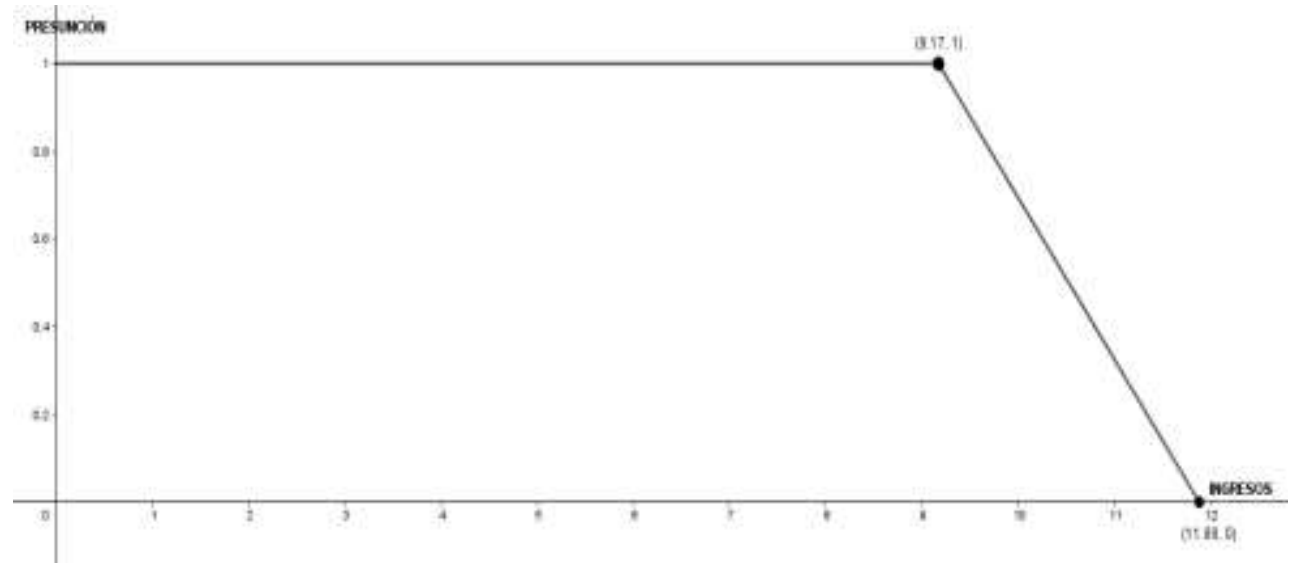

Gráfico 1. Estimación de Recursos económicos (unidades de mil)

Fuente: Elaboración propia

Los números borrosos trapezoidales (NBTr) denotados por ( $\left.\mathrm{a}_{1},\left[\mathrm{a}_{2}, \mathrm{a}_{3}\right], \mathrm{a}_{4}\right)$, expresan que, $\mathrm{a}_{1}$ = extremo inferior; $\left[a_{2}, a_{3}\right]$ = máximo de presunción; $a_{4}=$ extremo superior. Se presenta el grafico con lo explicado. 


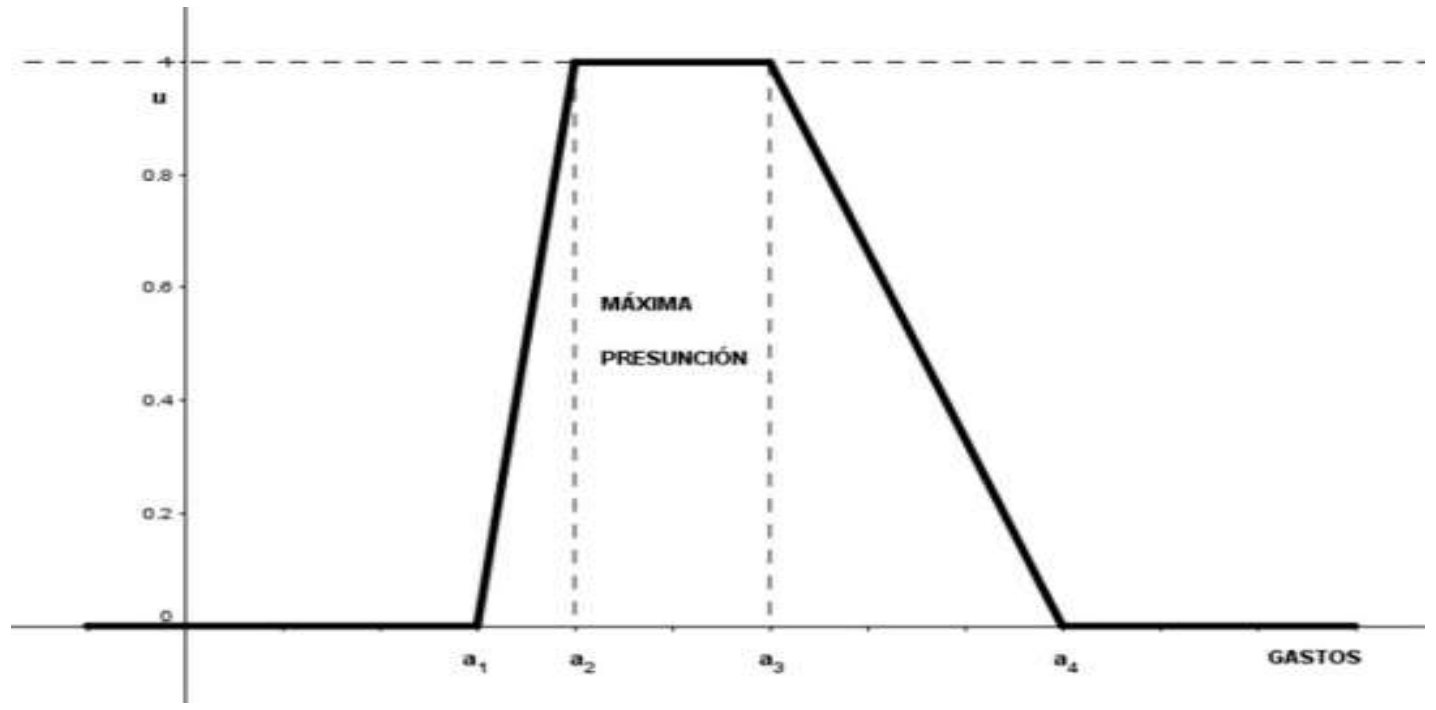

Gráfico 2. Número borroso trapezoidal (NBTr)

Fuente: Elaboración propia

Las áreas de trabajo de la empresa enunciados anteriormente, establece los niveles presupuestarios de acuerdo a la opinión de los expertos, con el propósito de llegar a alcanzar los objetivos establecidos. Estos niveles se presentan de la siguiente manera:

$\begin{array}{llcccc} & & \text { a1 } & \text { a2 } & \text { a3 } & \text { a3 } \\ \text { Nivel 1: } & \text { A0 } & 1.550 & 1.800 & 2.100 & 2.350 \\ \text { Nivel 2: } & \text { A0+A1 } & 2.350 & 2.600 & 2.900 & 3.150 \\ \text { Nivel 3: } & \mathrm{A} 0+\mathrm{A} 1+\mathrm{B} 0 & 5.850 & 6.100 & 6.400 & 6.650 \\ \text { Nivel 4: } & \mathrm{A} 0+\mathrm{A} 1+\mathrm{B} 0+\mathrm{C} 0 & 6.350 & 6.600 & 6.900 & 7.150 \\ \text { Nivel 5: } & \mathrm{A} 0+\mathrm{A} 1+\mathrm{B} 0+\mathrm{B} 1+\mathrm{C} 0 & 7.150 & 7.400 & 7.700 & 7.950 \\ \text { Nivel 6: } & \mathrm{A} 0+\mathrm{A} 1+\mathrm{B} 0+\mathrm{B} 1+\mathrm{B} 2+\mathrm{C} 0 & 7.650 & 7.900 & 8.200 & 8.450 \\ \text { Nivel 7: } & \mathrm{A} 0+\mathrm{A} 1+\mathrm{B} 0+\mathrm{B} 1+\mathrm{B} 2+\mathrm{C} 0+\mathrm{C} 1 & 9.350 & 9.600 & 9.900 & 10.150 \\ \text { Nivel 8: } & \mathrm{A} 0+\mathrm{A} 1+\mathrm{B} 0+\mathrm{B} 1+\mathrm{B} 2+\mathrm{C} 0+\mathrm{C} 1+\mathrm{D} 0 & 10.850 & 11.100 & 11.400 & 11.650 \\ \text { Nivel 9: } & \mathrm{A} 0+\mathrm{A} 1+\mathrm{B} 0+\mathrm{B} 1+\mathrm{B} 2+\mathrm{C} 0+\mathrm{C} 1+\mathrm{D} 0+\mathrm{D} 1 & 11.350 & 11.600 & 11.900 & 12.150 \\ \text { Nivel 10: } & \mathrm{A} 0+\mathrm{A} 1+\mathrm{B} 0+\mathrm{B} 1+\mathrm{B} 2+\mathrm{C} 0+\mathrm{C} 1+\mathrm{D} 0+\mathrm{D} 1+\mathrm{D} 2 & 12.350 & 12.600 & 12.900 & 13.150\end{array}$

En forma geométrica se presentan los diez niveles presupuestarios, inician desde el nivel 1 con una inversión de $(1.550,[1.800,2.100], 2.350)$ dólares, hasta el nivel 10 cuyo financiamiento será de $(13.150,[12.600,12.900], 13.150)$ dólares. Apoyados en los números borrosos trapezoidales (NBTr) se trazan trapezoides, estos representan niveles presupuestarios para cada objetivo planteado con su restricción presupuestaria. Lo indicado se aprecia en el siguiente gráfico. 


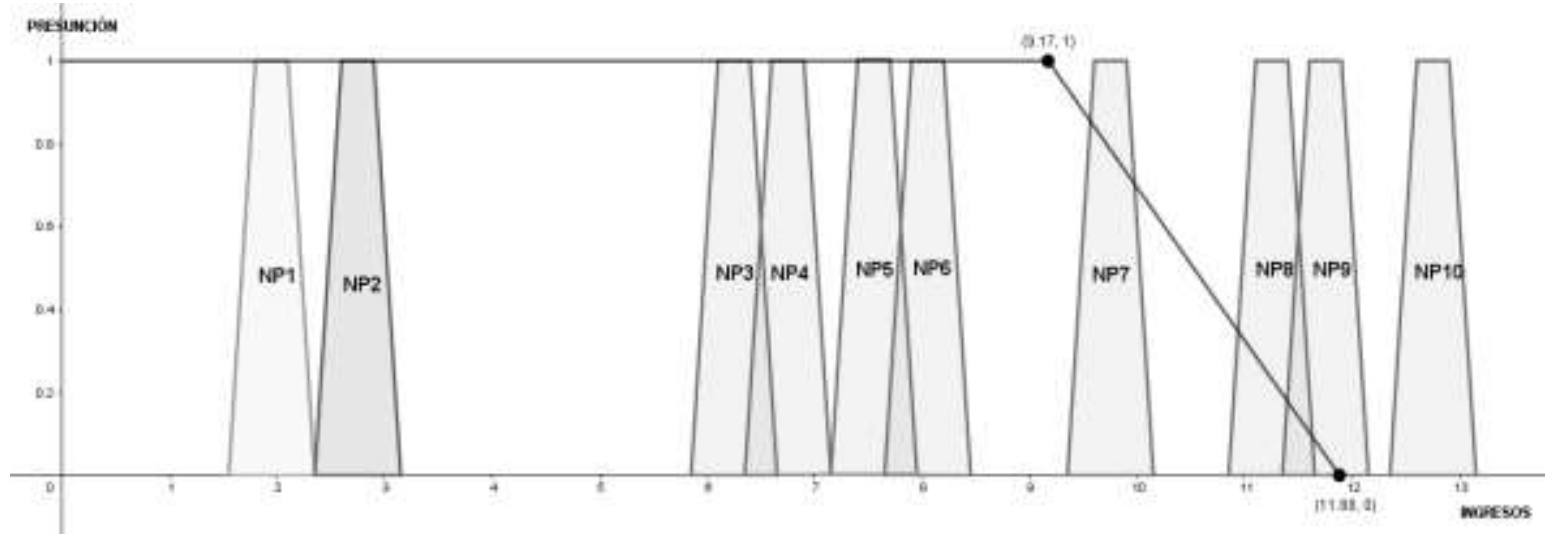

Gráfico 3. Niveles Presupuestados (unidades de mil)

Fuente: Elaboración propia

En el gráfico 3, los niveles del 1 al 6 son aprobados directamente, por lo que el presupuesto cubre los rubros asignados. La administración tendrá que analizar los demás rubros ya el existe falta de cobertura, con la finalidad de aceptar o rechazar.

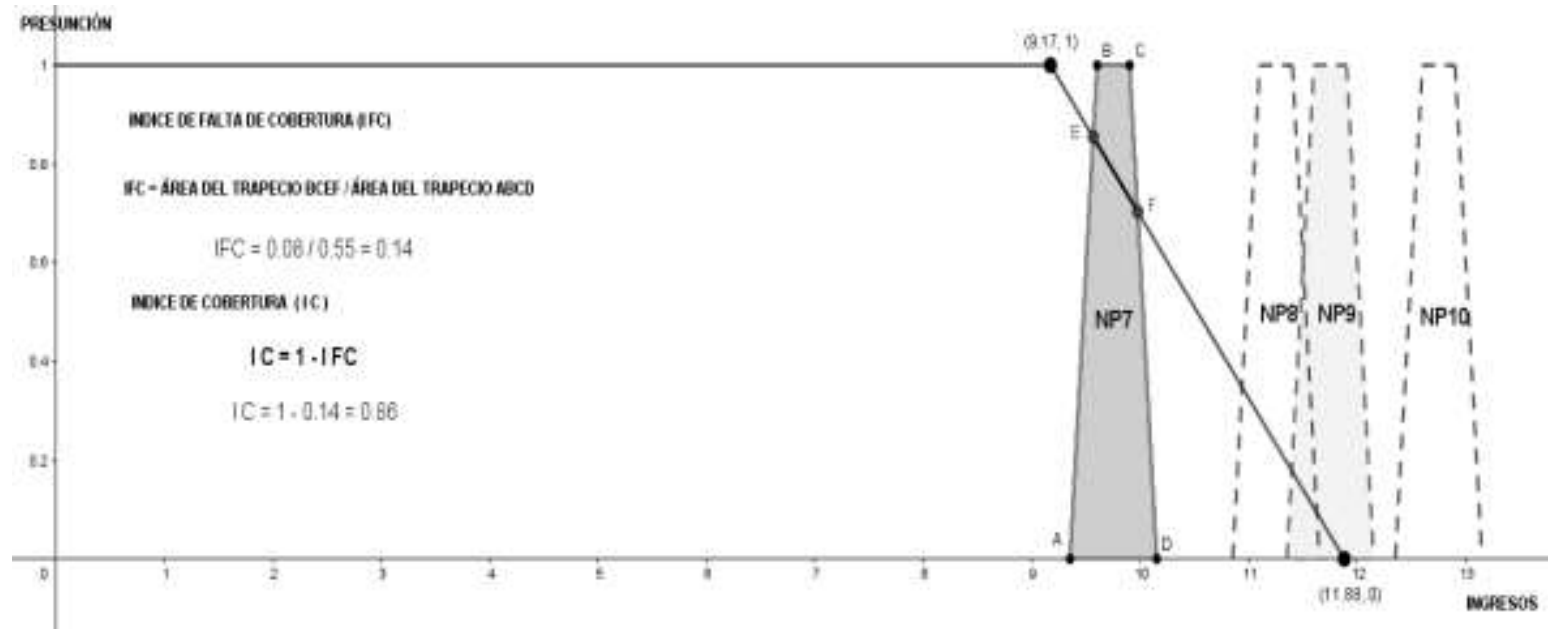

Gráfico 4. Análisis del Nivel Presupuestario 7 (unidades de mil)

Fuente: Elaboración propia

Geométricamente se obtiene el índice de falta de cobertura y su correspondiente complementario. Se obtiene el punto de intersección E y F a través de la intersección de las rectas que pasan por los puntos $\mathrm{AB}$ y $\mathrm{CD}$ respectivamente. Se procede a encontrar las áreas de los trapecios AEFD y ABCD, calculando el cociente entre ellos, al que se le denota como IFC (índice de falta de cobertura), su complemento IC = 1 - IFC llamado índice de cobertura, 
para el caso de estudio el nivel presupuestario 7 está cubierto en un $86 \%$, dejando la aprobación a nivel de administración.

Se procede de la misma manera a realizar el análisis del nivel presupuestario 8 , de acuerdo al gráfico 5 .

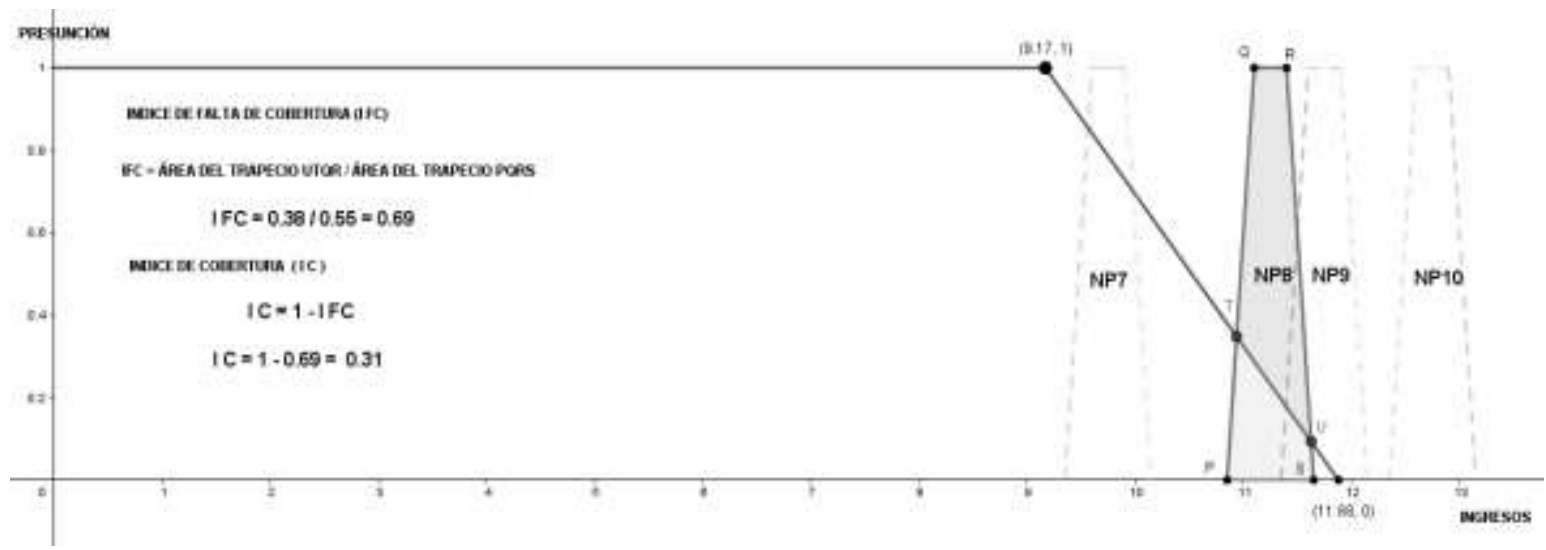

Figura 5. Análisis del Nivel Presupuestario 8 (unidades de mil)

Fuente: Elaboración propia

Siguiendo el procedimiento anterior, mediante el análisis geométrico para el nivel presupuestario 8, el índice de cobertura es del 31\%, la administración de la empresa analizará este porcentaje para su aprobación a su rechazo, dejando al mejor criterio la necesidad de aprobación ajustando el presupuesto a acciones complementarias. En relación a los niveles 9 y 10 , por su nivel de cobertura nulo deben ser rechazados directamente por la empresa, la decisión queda de que, si existe o no necesidad de cubrir estos niveles, sabiendo que el presupuesto no cubre el cumplimiento de estos objetivos.

\section{Resultados}

La presentación de este nuevo modelo de presupuesto, aplicando el método del Presupuesto Base Cero, y con el apoyo de la lógica difusa aplicando números borrosos trapezoidales (NBTr), se determinan los niveles presupuestarios mediante los cuales se cumplirán los objetivos trazados por las diferentes áreas de la empresa de muebles de madera, caso del presente estudio.

Desarrollando las herramientas de avanzada de la lógica borrosa, se determina una restricción presupuestaria entre el intervalo de 9.170,00 y 11.880,00 dólares, lo que significa que cualquier valor mayor a la perspectiva optimista existirá complejidad en el cumplimiento. 
Los niveles presupuestarios del 1 al 6, se encuentran dentro de la aceptación presupuestaria. El nivel 7 por tener un porcentaje representativo del $86 \%$ de cobertura, deberá ser aprobado para su ejecución. El nivel presupuestario 8, su índice de cobertura es del $31 \%$, este deberá ser analizado para su aprobación o rechazo ya que se hace difícil el cumplimiento de este nivel. Los niveles 9 y 10 cuyo índice de cobertura es nulo, deben ser rechazados ya que no se llegará a cumplir con sus objetivos entre ellos, Implementación de internet y software; e, Implementación de muebles y decoraciones.

\section{Conclusiones}

- La aplicación del presupuesto Base cero (PBC) con el apoyo de la lógica difusa, cumple dos grandes funciones. El primero se estructura en base al cumplimento de objetivos trazados, y no en relación a un historial de rubros económicos de años anteriores, con asignación de recursos económicos de manera eficiente con el propósito de cumplir con los niveles presupuestarios determinados. La segunda función de este nuevo modelo de presupuesto es la aplicación de números borrosos trapezoidales (NBTr), este permite reducir la incertidumbre dentro de los niveles presupuestarios establecidos, con la finalidad de lograr el cumplimiento de los objetivos, con esta herramienta se determinan los niveles que se encuentren dentro del índice de cobertura, el cual permitirá la aceptación directa por parte de la administración de la empresa.

- Este estudio presenta la aprobación de los niveles del 1 al 7, ya que alcanza los índices de cobertura, el nivel 8, tendrá que ser analizado por tener un índice de cobertura no aceptable, este mejoraría a medida que los ingresos generen un crecimiento; y, los dos últimos niveles por no estar dentro del índice de cobertura, no serán tomados en consideración. Este nuevo cálculo de presupuesto será entregado a los artesanos en la fabricación de muebles de madera del cantón Santiago de Méndez de la Provincia de Morona Santiago-Ecuador, con la finalidad de mejorar su gestión empresarial desde la óptica del porqué se debe invertir y no cuanto se debe invertir, con ello se mejoraría la toma de decisión a nivel gerencial.

\section{Referencias Bibliográficas}

Burbano, J. (2005). Presupuestos. Enfoques de gestión, planeación y control de recursos. Bogotá: McGraw-Hill Interamericana S.A.

Lazzari, L. (1997). Teoría de la decisión fuzzy. Buenos aires, Argentina: Editorial Macchi

Ciscomani, F. (2015). Presupuesto Base Cero: retos y perspectivas. El Cotidiano, 192, 5862. Recuperado de http://www.redalyc.org/articulo.oa?id=32539883007

Tovar, R. (2015). Presupuesto Base Cero: eficiencia y sesgos a la inercia. El Cotidiano,192, 33-36. Recuperado de http://www.redalyc.org/articulo.oa?id=32539883004 
Sánchez, M. (2015). Presupuesto Base Cero, una oportunidad para invertir mejor. El Cotidiano, 192, 85-92. $\quad$ Recuperado de http://www.redalyc.org/articulo.oa?id=32539883011

Pérez, H. (2015). Reflexiones sobre el Presupuesto Base Cero y el Presupuesto basado en Resultados. El Cotidiano, 192, 78-84. Recuperado de http://www.redalyc.org/articulo.oa?id=32539883010

Chávez, J. (2015). ¿Por qué Presupuesto Base Cero (PB0)? El Cotidiano, 192, 69-77. Recuperado de http://www.redalyc.org/articulo.oa?id=32539883009

Kaufmann, A. y Gil, J. (1986). Introducción de la teoría de subconjuntos borrosos a la gestión de las empresas. España: Milladoiro.

Kaufmann, A. y Gil, J. (1987). Técnicas operativas de gestión para el tratamiento de la incertidumbre. Hispano Europea, Barcelona.

Kaufmann, A., y Gil-Aluja, J. (1989). Modelos para la investigación de efectos olvidados. Barcelona, España: Milladoiro.

Lafuente, J. G. (1997). Marketing para el nuevo milenio: nuevas técnicas para la gestión comercial en la incertidumbre. Barcelona, España: Ediciones Pirámide.

Arango, M., Urán, C., y Pérez, G. (2012). La gestión de indicadores empresariales con lógica difusa para la toma de decisiones. Lámpsakos, 8, 47-53. Recuperado de https://dialnet.unirioja.es/servlet/articulo?codigo $=4490568$

Chalco, C., Pedraza, J., Román, H., y Álvarez, G. (2009). Una aproximación de números difusos de tipo trapezoidal. Ingeniare. Revista Chilena de Ingeniería, 17(2), 158-165. Recuperado de http://www.redalyc.org/articulo.oa?id=77211359004

Medina, S. (2006). Estado de la cuestión acerca del uso de la lógica difusa en problemas financieros. Cuadernos de Administración, XIX (32), 195-223. Recuperado de htt://cuadernosadministracion.javeriana.edu.co/pdfs/8 32 estado de la cuestión.pdf

Luna, K., Tinto, J., Sarmiento, W. y Cisneros, D. (2018). Implementación de un presupuesto empresarial base cero bajo el enfoque difuso. Revista Ciencia UNEMI, 11(27), 43 51. Recuperado de http://ojs.unemi.edu.ec/index.php/cienciaunemi/article/view/373

Reig, J. y González, J. (2002). Modelo borroso de control de gestión de materiales. Revista Española de Financiación y Contabilidad, 31(112), 431-459. Recuperado de https://www.jstor.org/stable/42781484

Zadeh, L. (1965). Fuzzy Sets. Information and Control, 8(4), 338-353. Recuperado de https://www.cambridge.org/core/journals/journal-of-symbolic-logic/article/div- 
classtitlezadehl-a-fuzzy-sets-information-and-control-vol-8-1965-pp-338353-

zadehl-a-similarity-relations-and-fuzzy-orderings-information-sciences-vol-3-1971pp-177200div/3A80F34917B4A88DD12299044FD1E47E

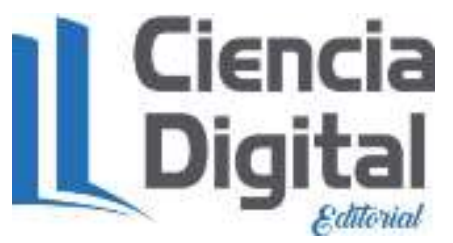


Para citar el artículo indexado.

Espinoza J., Luna K., Sarmiento W. \& Erazo J. (2019) Lógica borrosa como herramienta de apoyo en la elaboración de un presupuesto base cero: Caso artesanos de muebles de madera. Revista electrónica Ciencia Digital 3(2), 285-394. Recuperado desde: http://cienciadigital.org/revistacienciadigital2/index.php/CienciaDigital/article/view/411/938

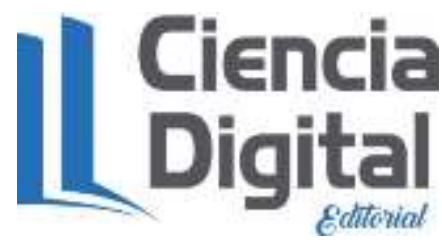

El artículo que se publica es de exclusiva responsabilidad de los autores y no necesariamente reflejan el pensamiento de la Revista Ciencia Digital.

El articulo queda en propiedad de la revista y, por tanto, su publicación parcial y/o total en otro medio tiene que ser autorizado por el director de la Revista Ciencia Digital.
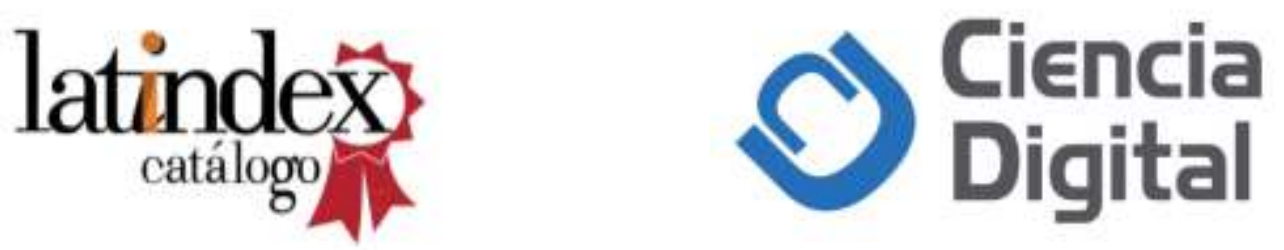